\title{
Short communication: Transcriptional response to a large genomic island deletion in the dairy starter culture Streptococcus thermophilus
}

\author{
Kurt Selle, ${ }^{1,2 *}$ Joakim M. Andersen, ${ }^{1}$ and Rodolphe Barrangou ${ }^{1,2 *}$ \\ ${ }^{1}$ Department of Food, Bioprocessing and Nutrition Sciences, North Carolina State University, Raleigh 27695 \\ ${ }^{2}$ Functional Genomics Graduate Program, North Carolina State University, Raleigh 27695
}

\section{ABSTRACT}

Streptococcus thermophilus is a lactic acid bacterium widely used in the syntrophic fermentation of milk into yogurt and cheese. Streptococcus thermophilus has adapted to ferment milk primarily through reductive genome evolution but also through acquisition of genes conferring proto-cooperation with Lactobacillus bulgaricus and efficient metabolism of milk macronutrients. Genomic analysis of Strep. thermophilus strains suggests that mobile genetic elements have contributed to genomic evolution through horizontal gene transfer and genomic plasticity. We previously used the endogenous type II CRISPR-Cas [clustered regularly interspaced short palindromic repeats (CRISPR) with CRISPR-associated sequences (Cas)] system in Strep. thermophilus to isolate derivatives lacking the chromosomal mobile genetic element and expandable island that display decreased fitness under routine culturing conditions. Of note, the Lac operon and Leloir pathway genes were deleted in the largest expendable genomic island (102 $\mathrm{kbp}$ ), rendering the strain incapable of acidifying milk. However, the removal of other open reading frames in the same island had unclear effects on the fitness and regulatory networks of Strep. thermophilus. To uncover the physiological basis for the observed phenotypic changes and underlying regulatory networks affected by deletion of the 102-kbp genomic island in Strep. thermophilus, we analyzed the transcriptome of the mutant that lacked $\sim 5 \%$ of its genome. In addition to the loss of transcripts encoded by the deleted material, we detected a total of 56 genes that were differentially expressed, primarily encompassing 10 select operons. Several predicted metabolic pathways were affected, including amino acid and purine metabolism, oligopeptide transport, and iron transport. Collectively, these results suggest that deletion of a 102-kb genomic island in Strep. thermophilus influences compensatory

Received January 29, 2019.

Accepted May 2, 2019.

*Corresponding authors: kmselle@ncsu.edu and rbarran@ncsu.edu transcription of starvation stress response genes and metabolic pathways involved in important niche-related adaptation.

Key words: fermentation, Streptococcus thermophilus, transcriptome, CRISPR

\section{Short Communication}

Comparative genomic analysis of Streptococcus thermophilus has revealed rapid and extensive genome evolution through purifying selection, as part of its adaptation to the dairy environment (Bolotin et al., 2004). Members of the Streptococcus salivarius subgroup have evolved toward commensalism by selective inactivation or loss of virulence genes associated with a pathogenic lifestyle in streptococci (Bolotin et al., 2004). Moreover, Strep. thermophilus acquired milk adaptation genes, which increased its fitness in the dairy environment, and shifted its regulatory networks, which facilitated proto-cooperation with Lactobacillus delbrueckii ssp. bulgaricus (Herve-Jimenez et al., 2009). As such, Strep. thermophilus is the only streptococcal species considered generally recognized as safe based on a long history of safe human use and consumption in fermented food products. Streptococcus thermophilus harbors a large number of insertion sequence elements that have contributed to its genomic evolution via gene accretion and deletion (Makarova et al., 2006). Thorough genomic comparisons have identified regions associated with insertion sequence elements that are strain-specific or exhibit polymorphism between Strep. thermophilus strains, revealing likely horizontal gene transfer (HGT) events (Bolotin et al., 2004; Goh et al., 2011). In some cases, HGT events between Strep. thermophilus and milk-adapted lactic acid bacteria or other streptococcal species are evident. For example, one genomic island (GEI) encoding proteinase prtS in Strep. thermophilus, and likely originating from Streptococcus suis, demonstrated a direct contribution of HGT to fitness in milk as measured by acidification rate (Dandoy et al., 2011). However, larger GEI often constitute "genetic sinks," encoding many acces- 
sory genes with unclear annotation or unknown function (Juhas et al., 2009). Consequently, known effects of GEI on host physiology in Strep. thermophilus has been limited to individual gene activities via reductive biology rather than total gene content en bloc. In our previous work, endogenous type II CRISPR-Cas [clustered regularly interspaced short palindromic repeats (CRISPR) with CRISPR-associated sequences (Cas)] counterselection enabled isolation of mutants lacking 4 GEI in Strep. thermophilus LMD-9 (Selle et al., 2015). Genomic sequencing was used to confirm the loss of the intervening sequence for the largest island (GEI4), encoding the Lac operon, which is responsible for the uptake and catabolism of lactose. As expected, deletion of the island resulted in conversion of Strep. thermophilus from a $\mathrm{Lac}^{+}$to a $\mathrm{Lac}^{-}$phenotype, but the deletion strain also exhibited reduced fitness compared with the wild-type parent (Supplemental Figure S1; https:// doi.org/10.3168/jds.2019-16397). Both the generation time and final optical density at $600 \mathrm{~nm}$ of the deletion mutant were significantly diminished under routine culture conditions in rich broth medium, but the basis for the observed phenotype remained unclear. To assess the biochemical and functional pathways this genomic island affects in Strep. thermophilus LMD-9, we investigated the transcriptional impact of GEI4 deletion by sequencing total RNA from the $\triangle$ GEI4 mutant and analyzed differential expression of genes compared with the wild-type parent.

A single replicate of each Strep. thermophilus strain was propagated in Elliker medium (Difco Laboratories, Detroit, MI) supplemented with $1 \%$ beef extract (wt/ vol) and $1.9 \%$ (wt/vol) $\beta$-glycerolphosphate (Sigma Chemical Co., St. Louis, MO) broth under static aerobic conditions at $37^{\circ} \mathrm{C}$. The Strep. thermophilus cultures were grown to a final optical density at $600 \mathrm{~nm}$ of 0.6 to 0.8 and centrifuged at $4,000 \times g$ for $10 \mathrm{~min}$ at $4^{\circ} \mathrm{C}$. The supernatants were decanted and cell pellets maintained at $-80^{\circ} \mathrm{C}$ until thawed with $1 \mathrm{~mL}$ of Tri-reagent using the Zymo Direct-zol RNA MiniPrep Kit (Zymo Research, Irvine, CA). Cells were then lysed using a mini bead beater on the homogenize setting for 5 cycles of $1 \mathrm{~min}$ of beating alternated with $1 \mathrm{~min}$ on ice. Total RNA was quantified with NanoDrop (Thermo Fisher Scientific, Waltham, MA) and analyzed for quality using an Agilent 2100 Bioanalyzer (Agilent Technologies, Santa Clara, CA). Library prep and RNA-sequencing were performed at the High-Throughput Sequencing and Genotyping Unit of the Roy J. Carver Biotechnology Center at the University of Illinois at UrbanaChampaign. For each sample, ribosomal RNA was removed with the Ribozero Bacteria Kit (Illumina Inc., San Diego, CA) followed by library preparation with the TruSeq Stranded RNA Sample Prep Kit (Illumina Inc.). Single-read RNA-sequencing (RNA-seq) was performed using an Illumina HiSeq 2500 Ultra-HighThroughput Sequencing system (Illumina Inc.) with a read length of $180 \mathrm{nt}$. Raw sequencing reads were assessed for quality using FastQC version 0.11.3 (http:// www.bioinformatics.babraham.ac.uk/projects/fastqc/) and processed using Geneious 8.0.5 (Kearse et al., 2012). Briefly, after adaptor sequences were trimmed, raw reads were quality-trimmed to remove sequence reads with an error probability limit of 0.001 (Phred score of 30) and filtered to remove reads shorter than 20 nt. These quality-trimmed and filtered sequences were then mapped onto the reference genomes using Bowtie 2 (Langmead and Salzburg, 2012) with default settings within Geneious 8.0.5 (Kearse et al., 2012). Expression was calculated in Geneious under the default settings, wherein reads mapped ambiguously were counted as partial reads. Expression of genes across samples was compared using the median of gene expression ratio normalization, also known as DE-Seq analysis (Dillies et al., 2013). The threshold $P$-value was calculated by performing a Bonferroni correction, treating each open reading frame (ORF) as an independent test.

Previously, we showed that CRISPR-targeting of GEI in Strep. thermophilus could result in screening and selection of low-incidence or low-frequency spontaneous mutants in mixed populations (Figure 1; Selle et al., 2015). A decrease in growth fitness in a semi-defined medium was observed for the $\Delta$ GEI4 strain. We aimed to assign and ascribe the fitness cost to select genes encoded on the island although it encompassed many unannotated and pseudo-genes. We therefore analyzed the expression profile during exponential growth of Strep. thermophilus by sequencing total RNA to gain transcriptional insights into the role of GEI4 in regulating Strep. thermophilus metabolism and physiology. The distribution of gene expression values was lower for GEI4 than for the rest of the genome within the wild-type parent (Figure 2B); however, the Lac operon and Leloir pathway genes were highly expressed (Supplemental Table S1; https://doi.org/10.3168/jds .2019-16397). In fact, even in the presence of sucrose, glucose, and lactose in the medium, lac $Z$ was one of the most highly expressed genes across the entire genome (Supplemental Table S1).

Next, we analyzed the transcriptional profile of $\Delta$ GEI4. Mapping the RNA-seq reads to the reference genome corroborated the loss of the intervening sequences for GEI4 generated by CRISPR-Cas selection (Figure 2A). Genes STER_1347 and STER_1348 exhibited some coverage within GEI4 in the deletion strain, but further inspection revealed an identical copy 
at $99.5 \%$ sequence identity of the genes at a separate locus (STER_0571 to STER_0572), leading to ambiguously mapped reads (Supplemental Figure S2; https:// doi.org/10.3168/jds.2019-16397). The DE-Seq analysis was performed to identify significantly differentially expressed (DE) genes, of which 56 were identified. A
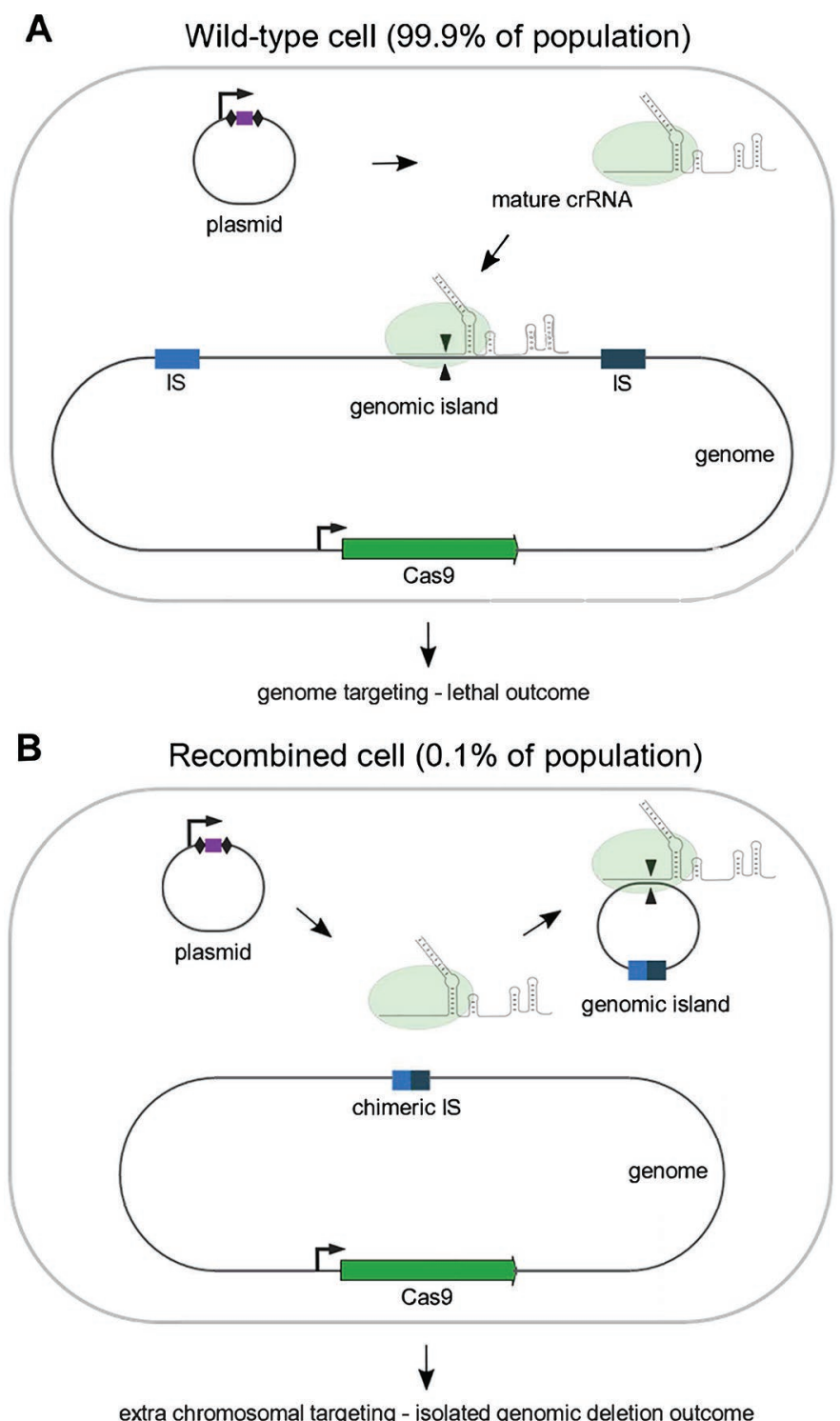

Figure 1. Schematic for recovery of isolated genomic deletion mutants by CRISPR-Cas [clustered regularly interspaced short palindromic repeats (CRISPR) with CRISPR-associated sequences (Cas)] selection. Routine propagation of Streptococcus thermophilus results in heterogeneous populations at certain loci due to recombination of insertion sequence elements (IS). Type II CRISPR (cr)RNA compatible with the endogenous Cas9 in Strep. thermophilus LMD-9 were designed to target lacZ and transformed into Strep. thermophilus. Wild-type cells (A) are effectively removed from the population due to genometargeting lethality, but cells that excise the genomic island (B) through recombination are not killed, instead losing the sequence via CRISPR degradation. total of 10 putative operons comprising 44 of the genes affected by deletion of GEI4 were observed (Table 1). Of these, 19 exhibited significant downregulation, affecting pathways related to aromatic amino acid metabolism (STER_1548 to STER_1555), sulfur amino acid metabolism (STER_0390 to STER_0393), stressrelated genes (STER_1821 to STER_1823) and cell envelope stress (STER_1960 to STER_1963). A total of 37 genes were upregulated in the absence of GEI4, which included operons of $\mathrm{ABC}$ oligopeptide transport (STER_1405 to STER_1409), purine biosynthesis (STER_0049to STER_0054), and iron binding and transport (STER_1020 to STER_1028).

Iron transport genes in Strep. thermophilus LMD9 are encoded by 2 colocalized operons: fat $A B C D$ (STER_1024 to STER_1028) and a second operon that includes a twin arginine translocase gene (STER_1020 to STER_1024), both of which were upregulated in the $\Delta$ GEI4 mutant. Typically, L. bulgaricus produces hydrogen peroxide during yogurt fermentation and, in response, Strep. thermophilus presumably downregulates iron binding and transport genes to minimize intracellular iron concentrations (Herve-Jimenez et al., 2009). It has been hypothesized (Herve-Jimenez et al., 2009) that Strep. thermophilus reduces expression of iron binding and transport genes in response to either hydrogen peroxide or L. bulgaricus to prevent lethal Fenton reaction products from accumulating. Moreover, the fatABCD operon in Strep. thermophilus is downregulated upon exposure to DNA damaging agents such as mitomycin $\mathrm{C}$ and norfloxacin (Boutry et al., 2013). It is noteworthy that the upregulation of the iron transport genes in $\triangle$ GEI4 occurs despite deletion of a glutamate-cysteine ligase (STER_1379) encoded on GEI4. The glutamate-cysteine ligase has been implicated in glutathione synthesis and protection against reactive oxygen species stress (Wang et al., 2017). Overall, it is perplexing that the mutant strain of Strep. thermophilus deficient in glutathione synthesis would exhibit upregulation of genes facilitating iron transport when it is likely more susceptible to oxidative stress generated by the internalization of iron. However, Strep. thermophilus also increases expression of iron transport genes during late-stage fermentation while still in the presence of $L$. bulgaricus with unknown causes or implications (Sieuwerts et al., 2010).

In the $\triangle$ GEI4 strain, the purine purCLFMNH operon (STER_0049 to STER_0054) was significantly upregulated (Table 1). This result is in contrast with the canonical response of Strep. thermophilus to coculture in milk with Lactobacillus delbrueckii ssp. bulgaricus, downregulating purine biosynthesis genes (Herve-Jimenez et al., 2009; Sieuwerts et al., 2010). It is hypothesized that L. delbrueckii ssp. bulgaricus 
A

LMD-9 genome
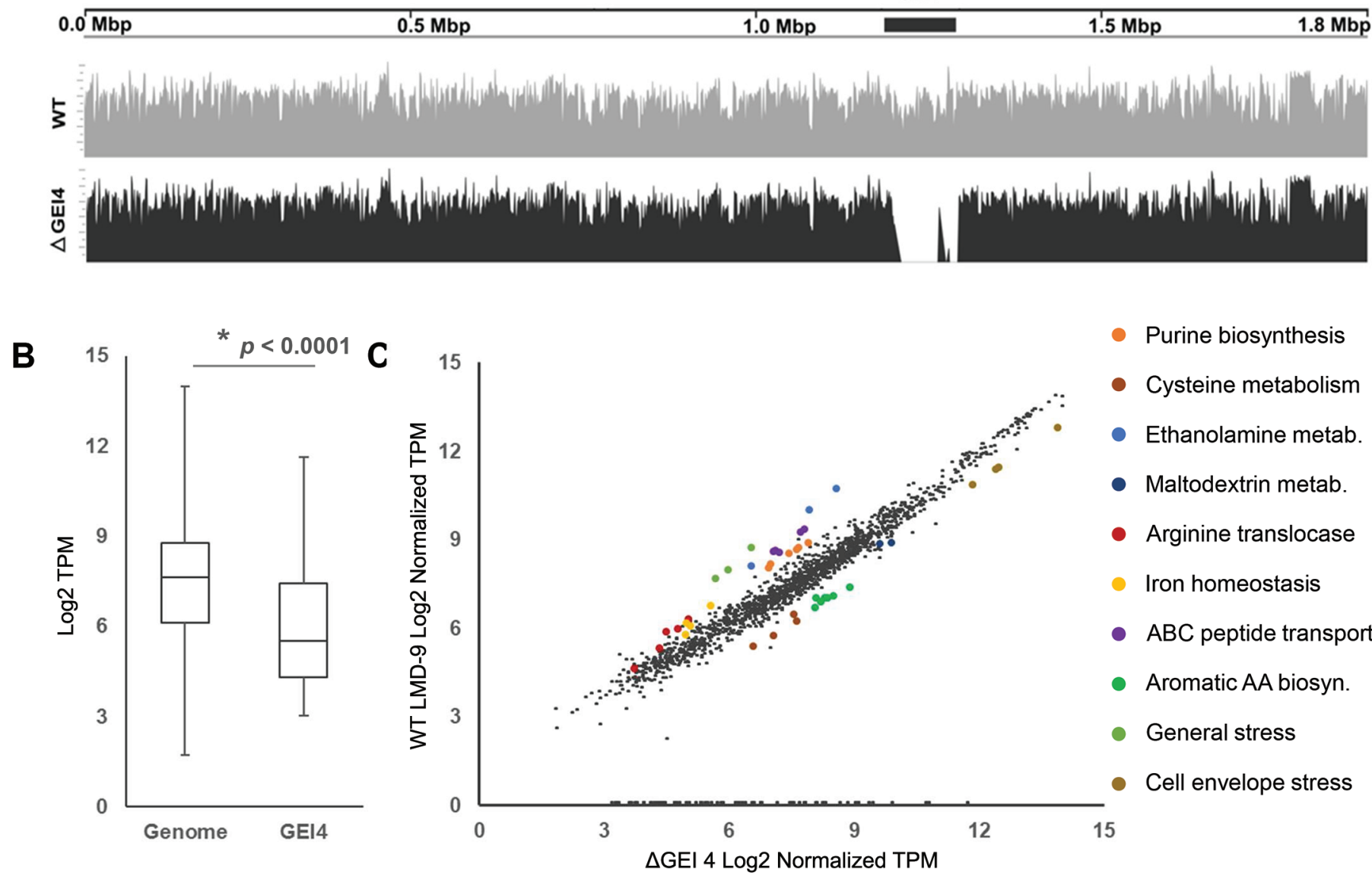

- Purine biosynthesis

- Cysteine metabolism

- Ethanolamine metab.

- Maltodextrin metab.

- Arginine translocase

- Iron homeostasis

- $\mathrm{ABC}$ peptide transport

- Aromatic AA biosyn.

- General stress

- Cell envelope stress

Figure 2. (A) RNA-sequencing read coverages for the wild-type parent (WT; top) and genomic island 4 deletion variant ( $\Delta$ GEI4; bottom) of Streptococcus thermophilus LMD-9. (B) Distribution of transcripts per million (TPM) gene expression values within the wild-type transcriptome ( $\mathrm{n}=1,866)$ compared with GEI4 $(\mathrm{n}=103)$. In the boxplot, the whiskers represent minimum and maximum values, the line across the middle of the box represents mean, and the box represents interquartile range. (C) Comparison of wild-type (NCK1125) and $\Delta$ GEI4 normalized TPM gene expression values. Differentially expressed genes are highlighted and colored according to operon affiliation.

provides nucleic acid intermediates to Strep. thermophilus during yogurt fermentation (Herve-Jimenez et al., 2009; Sieuwerts et al., 2010). A previous transcriptome study in Strep. thermophilus examined DE genes in a $\operatorname{cod} Y$ knockout strain and also showed upregulation of the purCLFMNH operon, suggesting that altered CodY activity could be responsible for regulation of the operon in opposition or addition to purR (Lu et al., 2015). CodY is a global transcriptional regulator of the stringent response in gram-positive bacteria, which typically senses intracellular branched-chain AA (BCAA) concentration. However, external signals affecting CodY activity that could result in transcriptional regulation of purCLFMNH are unknown. Interestingly, there are also putative xanthine permeases and a xanthine phosphoribosyltransferase contained within GEI4, but these genes are predicted to be degenerate by fragmentation or truncation.

Amino acid metabolism is highly significant to milk fermentation by Strep. thermophilus, which preferentially imports oligonucleotides from the protein-rich environment linked to the presence of extracellular protease. In order for ATP-binding cassette (ABC) and $о p p$ transporters to bind and import oligopeptides, extracellular surface proteinases process proteins into oligopeptide substrates. Typically, Strep. thermophilus strains internalize oligopeptides produced by L. bulgaricus through proteases such as PrtP (Settachaimongkon et al., 2014). In the case of Strep. thermophilus LMD-9 and SMQ-301, they encode their own cell surface-associated proteinase PrtS, which contributes to a fast acidification phenotype in industrial milk fermenta- 
$$
\text { (2. }
$$

$+$

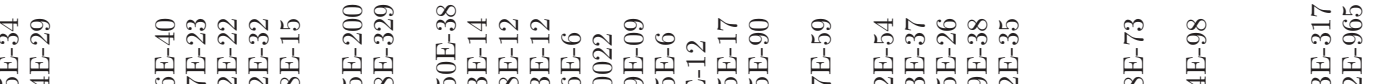
బ.

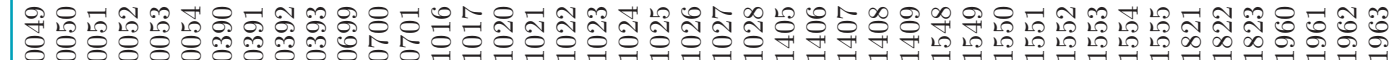

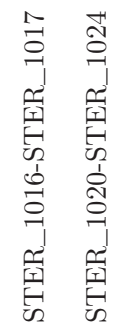

Oै

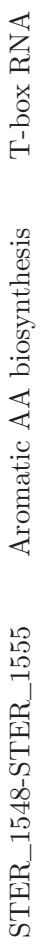


tion strains (Dandoy et al., 2011). One of the operons responsible for transport of oligopeptides in Strep. thermophilus, designated amiABCDE (STER_1405 to STER_1409), is upregulated in the $\triangle$ GEI4 strain. Of note, this operon is typically upregulated during coculture with $L$. delbrueckii ssp. bulgaricus in milk fermentation (Herve-Jimenez et al., 2008; Sieuwerts et al., 2010). Interestingly, the predicted regulator of the ami operon is CodY, based on the presence of a CodY binding motif, but knockout of $\operatorname{cod} Y$ has no effect on ami expression (Lu et al., 2015).

Operons responsible for biosynthesis of tryptophan (STER_1548 to STER_1555) and cysteine (STER_390 to STER_393) were downregulated in the $\Delta$ GEI4 strain. Similar to the purCLFMNH operon, CodY likely regulates the tryptophan biosynthesis operon, because it is downregulated upon knockout of CodY in Strep. thermophilus (Lu et al., 2015). CodY acts as a transcriptional repressor for AA metabolism during starvation, the effectors of which are GTP and BCAA. Interestingly, in starter cultures such as Lactococcus lactis and possibly Strep. thermophilus, it appears that $\operatorname{cod} Y$ is not regulated by GTP and is only known to respond to intracellular AA concentrations (Guédon et al., 2001; Lu et al., 2015). Previous work has demonstrated that the affinity of CodY for various promoter sequences may be influenced allosterically through the concentration of intracellular BCAA (Lu et al.., 2015). It is possible, therefore, that a gene encoded on GEI4 affects CodY activity by controlling the concentration of intracellular BCAA. Indeed, one of the most highly expressed genes on GEI4 under the conditions tested was annotated as a BCAA transport system II carrier protein (STER_1315). However, the function of this standalone gene is unknown and would need additional components to form a complete $\mathrm{ABC}$ transporter for BCAA.

It has previously been reported that the DNA damage response in Strep. thermophilus is counterregulated by the development of competence based on transcriptional responses to mitomycin C (Boutry et al., 2013). Previous work has shown that knockout of Strep. thermophilus LMD-9 DNA polymerase umuC1 (STER_1339), which happens to be encoded on GEI4, results in higher sensitivity to DNA damage-inducing agents such as norfloxacin and mitomycin C (Boutry et al., 2013). Moreover, the umuC1 gene is upregulated by knockout of the lexA-like repressor $h d i R$ and by addition of DNA damaging agents, highlighting its role in response to DNA damage. An hdiR binding site is located upstream of the umuC1 gene, but the relation of umuC1 gene knockout to the transcriptional changes observed here are unknown, given that none of the other genes regulated by HdiR were affected by GEI4 deletion. It is interesting to note that genes downregulated due to mitomycin C treatment (STER_0699 to STER_0701; STER_1025 to STER_1028; STER_1821 to STER_1823) are conversely upregulated in the $\triangle$ GEI4 strain.

To determine the relevance of operons identified here to the broader Strep. thermophilus species, we assessed the conservation of DE genes across 21 strains (Supplemental Table S2, Supplemental Figure S3; https:// doi.org/10.3168/jds.2019-16397). Operons encoding purine biosynthesis, cysteine metabolism, maltodextrin metabolism, ABC peptide transport, aromatic AA, and cell envelope stress were universally conserved across Strep. thermophilus strains ( $>90 \%$ nucleotide identity threshold). In contrast, ethanolamine metabolism, iron homeostasis, and general stress operons were still conserved but not ubiquitous across the 21 strains. These results suggest that several of the operons identified here could be broadly functional across Strep. thermophilus, warranting investigation into how these regulatory networks are integrated with GEI4 beyond strain LMD-9.

The results presented here stem from the advancement of next-generation genome editing and screening tools in bacteria, with whole-transcriptome profiling affording opportunities to delineate and determine the function of unknown or unclearly annotated genes encoded on chromosomal mobile genetic elements (Selle and Barrangou, 2015). Overall, our results indicate that the compensatory transcriptional changes due to GEI4 deletion have a material impact on the regulatory networks associated with important niche-related traits, such as AA and purine biosynthesis, iron metabolism, and potentially competence development. These data illustrate the contribution of HGT to the adaptation of Strep. thermophilus to milk and highlight a greater role for insertion sequence-encoded mobile genetic elements in coordinating bacterial metabolism and physiology. We envision that use of endogenous CRISPR-Cas systems in commensal and starter culture bacteria will enable further elucidation of the integration of mobile genetic elements with pathways important for fermentation and health-promoting characteristics of lactic acid bacteria (Sanozky-Dawes et al., 2015; van der Els et al., 2018).

\section{ACKNOWLEDGMENTS}

This work was funded in part by North Carolina Agricultural Foundation and DuPont Nutrition and Health (Wilmington, DE). The authors acknowledge the members of the Klaenhammer and Barrangou laboratory for helpful discussions. 


\section{REFERENCES}

Bolotin, A., B. Quinquis, P. Renault, A. Sorokin, S. D. Ehrlich, S. Kulakauskas, A. Lapidus, E. Goltsman, M. Mazur, G. D. Pusch, M. Fonstein, R. Overbeek, N. Kyprides, B. Purnelle, D. Prozzi, K. Ngui, D. Masuy, F. Hancy, S. Burteau, M. Boutry, J. Delcour, A. Goffeau, and P. Hols. 2004. Complete sequence and comparative genome analysis of the dairy bacterium Streptococcus thermophilus. Nat. Biotechnol. 22:1554-1558.

Boutry, C., B. Delplace, A. Clippe, L. Fontaine, and P. Hols. 2013. SOS response activation and competence development are antagonistic mechanisms in Streptococcus thermophilus. J. Bacteriol. 195:696-707. https://doi.org/10.1128/JB.01605-12.

Dandoy, D., C. Fremaux, M. H. de Frahan, P. Horvath, P. Boyaval, P. Hols, and L. Fontaine. 2011. The fast milk acidifying phenotype of Streptococcus thermophilus can be acquired by natural transformation of the genomic island encoding the cell-envelope proteinase PrtS. Microb. Cell Fact. 10(Suppl. 1):S21. https://doi.org/10 $.1186 / 1475-2859-10-$ S1-S21.

Dillies, M. A., A. Rau, J. Aubert, C. Hennequet-Antier, M. Jeanmougin, N. Servant, C. Keime, G. Marot, D. Castel, J. Estelle, G. Guernec, B. Jagla, L. Jouneau, D. Laloë, C. Le Gall, B. Schaëffer, S. Le Crom, M. Guedj, and F. Jaffrézic. 2013. A comprehensive evaluation of normalization methods for Illumina high-throughput RNA sequencing data analysis. Brief. Bioinform. 14:671-683. https://doi.org/10.1093/bib/bbs046.

Goh, Y. J., C. Goin, S. O'Flaherty, E. Altermann, and R. Hutkins. 2011. Specialized adaptation of a lactic acid bacterium to the milk environment: The comparative genomics of Streptococcus thermophilus LMD-9. Microb. Cell Fact. 10(Suppl. 1):S22. https://doi .org/10.1186/1475-2859-10-S1-S22.

Guédon, E., P. Serror, S. D. Ehrlich, P. Renault, and C. Delorme. 2001. Pleiotropic transcriptional repressor CodY senses the intracellular pool of branched-chain amino acids in Lactococcus lactis. Mol. Microbiol. 40:1227-1239. https://doi.org/10.1046/j.1365 $-2958.2001 .02470 . x$

Herve-Jimenez, L., I. Guillouard, E. Guedon, S. Boudebbouze, P. Hols, V. Monnet, E. Maguin, and F. Rul. 2009. Postgenomic analysis of Streptococcus thermophilus cocultivated in milk with Lactobacillus delbrueckii ssp. bulgaricus: Involvement of nitrogen, purine, and iron metabolism. Appl. Environ. Microbiol. 75:2062-2073.

Herve-Jimenez, L., I. Guillouard, E. Guedon, S. Boudebbouze, P. Hols, V. Monnet, F. Rul, and E. Maguin. 2008. Physiology of Streptococcus thermophilus during the late stage of milk fermentation with special regard to sulfur amino-acid metabolism. Proteomics 8:4273-4286. https://doi.org/10.1002/pmic.200700489.

Juhas, M., J. R. van der Meer, M. Gaillard, R. M. Harding, D. W. Hood, and D. W. Crook. 2009. Genomic islands: Tools of bacterial horizontal gene transfer and evolution. FEMS Microbiol. Rev. 33:376-393. https://doi.org/10.1111/j.1574-6976.2008.00136.x.

Kearse, M., R. Moir, A. Wilson, S. Stones-Havas, M. Cheung, S. Sturrock, S. Buxton, A. Cooper, S. Markowitz, C. Duran, T. Thierer, B. Ashton, P. Meintjes, and A. Drummond. 2012. Geneious Basic: An integrated and extendable desktop software platform for the organization and analysis of sequence data. Bioinformatics 28:1647-1649. https://doi.org/10.1093/bioinformatics/bts199.
Langmead, B., and S. L. Salzberg. 2012. Fast gapped-read alignment with Bowtie 2. Nat. Methods 9:357-359. https://doi.org/10.1038/ nmeth.1923.

Lu, W. W., Y. Wang, T. Wang, and J. Kong. 2015. The global regulator CodY in Streptococcus thermophilus controls the metabolic network for escalating growth in the milk environment. Appl. Environ. Microbiol. 81:2349-2358. https://doi.org/10.1128/AEM $.03361-14$

Makarova, K., A. Slesarev, Y. Wolf, A. Sorokin, B. Mirkin, E. Koonin, A. Pavlov, N. Pavlova, V. Karamychev, N. Polouchine, V. Shakhova, I. Grigoriev, Y. Lou, D. Rohksar, S. Lucas, K. Huang, D. M. Goodstein, T. Hawkins, V. Plengvidhya, D. Welker, J. Hughes, Y. Goh, A. Benson, K. Baldwin, J. H. Lee, I. Díaz-Muñiz, B. Dosti, V. Smeianov, W. Wechter, R. Barabote, G. Lorca, E. Altermann, R. Barrangou, B. Ganesan, Y. Xie, H. Rawsthorne, D. Tamir, C. Parker, F. Breidt, J. Broadbent, R. Hutkins, D. O'Sullivan, J. Steele, G. Unlu, M. Saier, T. Klaenhammer, P. Richardson, S. Kozyavkin, B. Weimer, and D. Mills. 2006. Comparative genomics of the lactic acid bacteria. Proc. Natl. Acad. Sci. USA 103:15611-15616.

Sanozky-Dawes, R., K. Selle, S. O'Flaherty, T. Klaenhammer, and R. Barrangou. 2015. Occurrence and activity of a type II CRISPRCas system in Lactobacillus gasseri. Microbiology 161:1752-1761. https://doi.org/10.1099/mic.0.000129.

Selle, K., and R. Barrangou. 2015. Harnessing CRISPR-Cas systems for bacterial genome editing. Trends Microbiol. 23:225-32. https:/ /doi.org/10.1016/j.tim.2015.01.008.

Selle, K., T. R. Klaenhammer, and R. Barrangou. 2015. CRISPRbased screening of genomic island excision events in bacteria. Proc. Natl. Acad. Sci. USA 112:8076-8081. https://doi.org/10.1073/ pnas. 1508525112 .

Settachaimongkon, S., M. J. Nout, E. C. Antunes-Fernandes, K. A. Hettinga, J. M. Vervoort, T. C. van Hooijdonk, M. H. Zwietering, E. J. Smid, and H. J. van Valenberg. 2014. Influence of different proteolytic strains of Streptococcus thermophilus in co-culture with Lactobacillus delbrueckii ssp. bulgaricus on the metabolite profile of set-yoghurt. Int. J. Food Microbiol. 177:29-36. https://doi.org/ 10.1016/j.ijfoodmicro.2014.02.008.

Sieuwerts, S., D. Molenaar, S.A. van Hijum, M. Beerthuyzen, M. J. Stevens, P. W. Janssen, and C. J. Ingham., deBok, F. A., W. M. de Vos, and J. E. van Hylckama Vlieg. 2010. Mixed-culture transcriptome analysis reveals the molecular basis of mixed-culture growth in Streptococcus thermophilus and Lactobacillus bulgaricus. Appl. Environ. Microbiol. 76:7775-7784.

van der Els, S., J. K. James, M. Kleerebezem, and P. A. Bron. 2018. Versatile Cas9-driven subpopulation selection toolbox for Lactococcus lactis. Appl. Environ. Microbiol. 84:e02752e17. https://doi .org/10.1128/AEM.02752-17.

Wang, Y., H. Y. He, H. H. Li, W. W. Lu, T. T. Guo, and J. Kong, 2017. The global regulator CodY responds to oxidative stress by the regulation of glutathione biosynthesis in Streptococcus thermophilus. J. Dairy Sci. 100:8768-8775. https://doi.org/10.3168/ jds.2017-13007. 